\title{
Far-sighted vision
}

\author{
US astronomers' latest list of priorities holds valuable lessons for other scientific disciplines.
}

\begin{abstract}
n 1964, a flood of post-Sputnik funding led US astronomers to draw up a list of large projects that could benefit the field. The result was the first decadal survey, a ranked wish list of new telescopes to build over the next ten years. The survey was an instant hit with politicians and funders, and similar priority lists are today drawn up in a range of disciplines.

But astronomy's decadal reviews have run into trouble of late, with overambitious lists, and projects busting their budgets and their schedules. The latest survey, published last week (see page 910), seeks to correct some of the fundamental problems with the previous reviews - and scientists in other fields drawing up their own wish lists would be well advised to take note.

The past few decadal surveys made the mistake of trying to appease all of astronomy's subdisciplines. Recent lists have, for example, included purpose-built instruments for fields such as X-ray and infrared astronomy, without assessing whether everything can actually be built in the decade at hand. At the same time, individual projects have grown in size and complexity, causing delays and spiralling costs. These problems have slowed each decade's agenda and have damaged the survey's credibility with the funding bodies it is meant to entice.

This year's survey aims to address these flaws while facing up to the realities of the ongoing financial crisis. It seeks to satisfy several subfields at once by endorsing large, flexible telescopes. In space, the panel's top priority is a wide-field infrared survey telescope that will hunt for extrasolar planets, study galaxy formation and measure dark energy - the mysterious force accelerating cosmic expansion.
\end{abstract}

\section{After the pandemic}

\section{Despite some mistakes, the World Health Organization handled the flu outbreak well.}

arlier this month, Margaret Chan, director-general of the World Health Organization (WHO) in Geneva, confirmed two things that many scientists already knew to be true: the H1N1 influenza pandemic is over, and the world was lucky. A disease that could have mutated into a highly lethal strain turned out to be comparatively mild.

Chan also reiterated that the WHO needs to review its handling of the pandemic. It should certainly revisit its decision to grant anonymity to the experts who sat on its emergency committee, and who wielded huge control over the decision to announce a global publichealth emergency. The WHO insists that anonymity was necessary to prevent committee members from being influenced. But it helped to fuel accusations that members from industry were just trying to sell more vaccine. In retrospect, openness would have tempered that criticism.
On the ground, a second wide-field telescope will image the entire visible sky every three nights, generating petabytes of data that will be accessible to astronomers all over the world.

The survey also attempts to bring more credibility to cost estimates by providing independent assessments of each project's budget. This approach is a hard lesson learned from the 2001 decadal review, whose top priority, the James Webb Space Telescope, has more than quadrupled in price.

The new strategy is certainly open to criticism. For one thing, there is a limit to how much astronomy's many subdisciplines can share an X-ray telescope is very different from an infrared telescope, for example. It is also unclear how deep the commitment to cooperation runs: the wide-field space telescope is a rebranding of a previous dark-energy mission, and those outside the field are suspicious that their work will be a lower priority than the original purpose. And the scrupulous budgeting means that some daring projects now receive lower priority than more conservative proposals.

The panellists hope to address these potential flaws with a more flexible decadal process. Rather than treating their document as carved in stone until 2020, the researchers call for a standing committee to carry out periodic reassessments of projects, along with a review of the progress in various priority areas, such as planet hunting. Both can change depending on how current and prospective projects perform.

The latest survey has clearly rescued the decadal process from torpidity. The list is relevant and affordable. Other disciplines planning their own reviews should follow its lead, as it promises to be a steady guide for a bumpy decade ahead.

The WHO finally released the names of the committee members on 11 August. And, as critics alleged, several of them have links to industry. But it would be shocking if they did not. Pharmaceutical companies play a key role in the response to pandemics, and they control a wealth of research knowledge on drugs and vaccines. But for the WHO to withhold their names until now caused unnecessary self-inflicted damage.

This should not distract from the post-pandemic verdict that the emergency committee, the WHO and its allied national health agencies around the world deserve praise - and our thanks. Throughout the crisis, they generally walked the difficult line between hype and dangerous understatement with aplomb. Although vaccines proved hard to make and their distribution could have been improved, the level of coordination between various bodies and researchers is heartening.

Of course, mistakes were made. But those who handled the world's response to H1N1 deserve better than false accusations of industry self-interest. Governments, industry and academics worked well together when faced with a potentially disastrous threat - and will hopefully do so when called upon again. 